\title{
INFRARED IMAGE SEGMENTATION BASED ON LOCAL STATISTICAL ACTIVE CONTOUR MODEL
}

\author{
Qi Hu ${ }^{a, b}$, Shuang Zhang, ${ }^{a, b}$ Xiaofeng Dong, ${ }^{a, b}$, Xiuyi Sun ${ }^{a, b}$, \\ Xinwen Wanga,b and Shuaiqi Liu ${ }^{a, b}$ \\ ${ }^{a}$ College of Electronic and Information Engineering, Hebei University, Baoding \\ 071000, P. R. China \\ ${ }^{b}$ Machine Vision Engineering Research Center of Hebei Province, Baoding 071000, \\ P. R. China
}

\begin{abstract}
Infrared image is widely used in medicine, military field and people's daily life. Infrared image segmentation is the basis of infrared image processing. In order to analyze other infrared images better, we propose a new segmentation algorithm of infrared image based on local statistical active contour and mathematical morphology. Firstly, the infrared image is processed to enhance the edge and smooth noise of the image; secondly, a novel locally statistical active contour model (ACM) is applied to infrared image segmentation; finally, we combine with mathematical morphology method to study the target and interference body adhesion problem of infrared image in the segmentation. The experiments show that this algorithm can be effectively used for infrared image segmentation.
\end{abstract}

Keywords: level set, infrared image segmentation, local statistical active countour.

\footnotetext{
${ }^{*}$ Corresponding author.

E-mail address: shdkj-1918@163.com (Shuaiqi Liu).
}

Copyright (C) 2017 Scientific Advances Publishers

2010 Mathematics Subject Classification: 80.

Submitted by Jianqiang Gao.

Received November 24, 2017 


\section{Introduction}

In many areas of research on infrared image processing, segmentation is a basic technology of infrared image. It can be applied to medical image processing, remote sensing satellite image processing, atmospheric condition prediction analysis, video monitoring image extraction, somatosensory technology of human identification, robot navigation and other fields.

Image segmentation is an important basic process in many computer vision and pattern recognition applications. It is the technique and process of dividing the image into several regions with different features and selecting the regions of interest. It is the key step from image processing to image target analysis [1]. At present, many promising new methods have been proposed for image segmentation, such as region based merging methods [1], graph based methods [2, 3], and active contour model based methods [4]. The active contour model mainly drives the curve to the boundaries of the segmented object to segment the image. The driving force mainly comes from the image data, including the edge based data or the region based force [4]. The edge based active contour model often uses local image gradient information to build some stop function, so as to drive the contour to stop at the boundary of the object. But the active contour model based on region aims at driving curve to evolve through some descriptor based on region [5]. The edge based active contour model method is suitable for images with intensity inhomogeneity. But generally speaking, they are sensitive to initialization of level set function. Moreover, they are vulnerable to severe boundary leakage for images with weak boundaries. Many regions based active contour models are based on global region information. They assume that the image intensity is uniform and therefore it is not suitable for segmenting images with intensity inhomogeneity. 
Accurate segmentation of gray inhomogeneous images is often a challenging task, because most of the algorithms are based on the strong symmetry of objects. It proposed a novel statistical local active contour model for image segmentation of inhomogeneous gray in [5]. Inhomogeneous objects are modelled as Gauss distributions for different mean and variance. The moving window is used to map the original image to another domain, where the intensity distribution of an inhomogeneous object is still Gauss, but can be better separated. The mean value of the Gauss distribution in the transform domain can be adaptively estimated by multiplying the bias field with the original signal in the window. Then, the statistical energy function is defined for each local region with the level set function and the constant of the real signal of the corresponding object according to the bias field. It is robust to initialization of the level setting function, allowing automatic application. This segmentation method will facilitate the segmentation and synthesis of existing images.

For segmenting objects in infrared images, due to the complexity of the edge of the target and the high mobility of the target state, the current research results still cannot achieve the engineering realization in the infrared image detection and tracking; in addition, the target will often release different forms of interference. It makes the gray feature of the target change and there will be a long time of occlusion and adhesion phenomenon, seriously affecting the accuracy of target detection and tracking. The complete occlusion cannot be solved only by means of image processing and it must be solved better by the research results in other fields. In order to segment the infrared image better and extract the target effectively, we apply the ACM algorithm proposed in [5] to infrared image segmentation and the mathematical morphology algorithm to solve the occlusion and adhesion problems in infrared image. In this paper, an infrared image segmentation algorithm based on novel local statistical active contour and mathematical morphology is proposed in order to segment infrared target better. 


\section{Infrared Image Preprocessing}

Histogram equalization is a method of treatment of modifying histogram. It is based on cumulative distribution function to extend gray values image of non-uniform distribution to gray level distribution image of uniform probability density. This method plays a very important role to binarization threshold selection. To the infrared gray image, the gray distribution of histogram is more concentrated. It can enhance the image features through the histogram equalization, increasing the overall contrast. This facilitates subsequent analysis and segmentation. However, the histogram equalization can make the noise prominent and the visual effect of the image reduced. So, it is necessary to use the appropriate de-noising algorithm to smooth the infrared image. Median filter is a nonlinear method. It is replaced a value with the median value of each sample in its neighbourhood of a digital image, in order to achieve the purpose of eliminating isolated noise. The speed of median filter is fast and the effect of image smoothing is better. Therefore, median filter is used in this paper to de-noise the image.

\section{Infrared Image Segmentation Based on Local Statistical Active Contour Model}

\subsection{A local statistical active contour model based on intensity inhomogeneous statistics}

The image segmentation algorithm based on level set is obtained mainly by minimizing energy function. And the energy function depends on the gradient of image and the model of image. Let $\Omega$ be the image domain, $b(x): \Omega \rightarrow R$ be the unknown bias field, $I(x): \Omega \rightarrow R$ be the

given image, $J(x): \Omega \rightarrow R$ be the real signal to be recovered, and $n(x): \Omega \rightarrow R$ be the noise. We consider the following intensity inhomogeneity model [6]:

$$
I(x)=b(x) J(x)+n(x) .
$$


We assume that there are $N$ objects in the image domain $\Omega$ and let $\Omega_{i}$ represent the domain of the $i$-th object. The real signal $J(x)$ to be restored is usually assumed to be a piecewise constant in each object domain, i.e., for $x \in \Omega_{i}, J(x)=c_{i}$, which $c_{i}$ is constant. The bias field $b$ is usually assumed to be smooth in the image domain $\Omega$. Assuming that the noise $n$ is Gauss distribution, it has zero mean and variance $\sigma_{n}^{2}$. Thus, the image intensity can be approximated by a Gauss distribution with mean $\mu_{n}$ and variance $\sigma_{n}^{2}$. However, the use of only one Gauss model is not accurate enough to describe the statistical properties of image intensities. Typically, we use multiple Gauss probability distributions to model image intensity and each Gauss probability distribution simulates the image intensity in each object domain. The distribution corresponding to the object domain $\Omega_{i}$ is

$$
p\left(I(y) \mid \theta_{i}\right)=\frac{1}{\sqrt{2 \pi} \sigma_{i}} \exp \left(-\frac{\left(I(y)-\mu_{i}(x)\right)^{2}}{2 \sigma_{i}^{2}}\right),
$$

where $\mu_{i}(x)$ is the average of the spatial variation and $\sigma_{i}$ is the standard deviation. Because $b(x)$ changes slowly, we can assume that it is a constant in a small window. So, we can assume $\mu_{i}(x) \approx b(x) c_{i}$. In the following discussion, we can represent it by $\theta_{i}=\left\{c_{i}, \sigma_{i}, b\right\}$, and $\theta=\left\{\theta_{i}, i=1, \ldots, N\right\}$.

\subsection{Energy function construction}

For each position $x$ in the image domain, we use $O_{x}$ to represent the neighbourhood region center, i.e., $O_{x}=\{y \mid\|y-x\| \leq \rho\}$, where $\rho$ represents the radius of the region $O_{x}$. Since there are $N$ nonoverlapping objects in the image, where $\Omega_{i}$ is the domain of the $i$-th object, the whole image domain can be represented as $\Omega$, where $\Omega_{i} \cap \Omega_{j} \neq \varnothing, \forall i \neq j$. We define the mapping $T: I\left(x \mid \theta_{i}\right) \rightarrow I\left(x \mid \theta_{i}\right)$ from the original image intensity domain $D(T)$ to another domain $R(T)$ as follow: 


$$
I\left(x \mid \theta_{i}\right)=\frac{1}{m_{i}(x)} \sum_{y \in \Omega_{i} \cap O_{x}} I\left(x \mid \theta_{i}\right),
$$

where $m_{i}(x)=\left\|\Omega_{i} \cap O_{x}\right\|$. We assume that the intensity of pixels $x$ is independent [7]. Accordingly, $\forall I\left(x \mid \theta_{i}\right) \in R(T)$. The corresponding probability density function (PDF) remains the Gauss distribution, i.e., $I\left(x \mid \theta_{i}\right) \sim N\left(\mu_{i}, \sigma_{i}^{2} / m_{i}(x)\right)$.

Since the intensity inhomogeneity shows a smooth intensity change in the image, it can be assumed that $I\left(y \mid \theta_{i}\right) \approx I\left(x \mid \theta_{i}\right), \forall y \in \Omega_{i} \cap O_{x}$. Because the product of Gauss PDF is still Gauss distribution, there are

$$
\prod_{y \in \Omega_{i} \cap \Omega_{x}} p\left(I\left(y \mid \theta_{i}\right)\right) \approx p\left(I\left(x \mid \theta_{i}\right)\right)^{m_{i}(x)} \propto N\left(\mu_{i}, \frac{\sigma_{i}^{2}}{m_{i}(x)}\right) .
$$

Let $D=\left\{I\left(x \mid \theta_{i}\right), x \in \Omega, i=1, \ldots, N\right\}$, then we can obtain the likelihood function of the $i$-th object:

$$
p\left(D \mid \theta_{i}\right)=\prod_{x \in \Omega} p\left(I\left(x \mid \theta_{i}\right)\right) .
$$

The following joint likelihood function is constructed as follow:

$$
p(D \mid \theta)=\prod_{i=1}^{N} p\left(D \mid \theta_{i}\right)=\prod_{i=1}^{N} \prod_{x \in \Omega} p\left(I\left(x \mid \theta_{i}\right)\right)=\prod_{x \in \Omega} q(I(x \mid \theta)) .
$$

Here, $\theta=\left\{\theta_{i}, i=1, \ldots, N\right\}$, and

$$
q(I(x \mid \theta))=\prod_{i=1}^{N} p\left(I\left(x \mid \theta_{i}\right)\right) \approx \prod_{i=1}^{N} \prod_{x \in \Omega_{i} \cap \Omega_{x}} p\left(I\left(y \mid \theta_{i}\right)\right) .
$$

When the formula (4) is substituted into formula (7), the formula (7) becomes a single variable Gauss distribution. In addition, it can be seen from Equation (3) that the intensity in transform domain uses the information of adjacent pixels belonging to the same category. So the classification results are less sensitive to noise and can produce smoother boundary objects. 
We define the energy function $l(\theta)$ as a logarithmic likelihood function w.r.t. $p(D \mid \theta)$ :

$$
l(\theta)=-\log p(D \mid \theta)=\text { constant }-\sum_{i=1}^{N} \int_{\Omega} \int_{\Omega_{i} \cap \Omega_{x}} \log \left(p\left(I\left(y \mid \theta_{i}\right)\right)\right) d y d x .
$$

The model can be solved by four phase model alternating iterative algorithm. In detail, please refer to [5].

\subsection{Infrared image segmentation based on local statistical active contour model.}

The image obtained by image segmentation will show the condition of the conglutination between the target and the interference object, which is not conducive to information extraction or feature processing of the object. So we need to process the image further and separate the target from the interfering body. In view of this problem, this paper chooses mathematical morphology method. Not only can obtain better segmentation results, but also the processing is simple. Firstly, the mathematical morphology method is used to etch the image so as to eliminate all the boundary points of the object. But for each etching, the original two value image will be reduced by one circle. Expansion and corrosion are two opposing processes, so the swelling of the treated nuclei can be restored to the original image.

Although the corrosion operation can separate target adhesion part and the expansion operation can connect the disconnected image regions, there is a common problem in the two processes: after corrosion treatment, the area of the target image will be smaller than the area before processing and after expansion operation, the area of the target image will be greater than the area before. Therefore, when dealing with conglutination targets, we use the open operation and closed operation.

First, we start the open operation, i.e., the image is corroded first and then expanded. By open operation, we achieve the purpose of breaking the adhesion target and it can basically keep the original target size not change. Then we start the closed operation, i.e., the image is expanded 
first and then corroded. The closed operation is used to fill the small holes in the target area, connect the target object with similar distance and smooth the target boundary. It does not change the area of the part of the target object obviously.

In combination with the above ACM and morphological methods, a novel infrared image segmentation algorithm based on local statistical active contour model and mathematical morphology is proposed in this paper. The steps of the algorithm are as follows:

(1) The histogram equalization algorithm is used to enhance the original infrared image $I$, and the enhanced image $I_{e n}$ is obtained.

(2) The image $I_{e n}$ is segmented by using the infrared image segmentation algorithm based on the local statistical active contour model, and the segmented image $I_{\text {sep }}$ is obtained.

(3) The image $I_{\text {sep }}$ is opened and closed for twice with open and close operations, and the final segmentation image is obtained.

\section{Experimental Results and Analysis}

In this paper, the model parameters are initialized to $\tilde{b}=1, \widetilde{\sigma}_{i}=i$, $i=1, \ldots, N$, and set the time step of the level set to $\Delta t=1$ and the time step of regularization to $\Delta t_{2}=0.1$. We set up $\Delta t_{2}=0.01, \varepsilon=1, \rho=6$ in all experimental settings.

Figure 1 is the infrared image segmentation effect figure of the back of a man's hand. Figure 1(a) is the original infrared image. It can obviously see the boundary gray transition between target vein and background. After image preprocessing, we can obtain Figure 1(b). Although the infrared image pretreatment significantly increases the discrimination of target and background, the inhomogeneous phenomenon of the gray still cannot be overcome, which increases the difficulty of target segmentation. However, as shown in Figure 1(c), the algorithm can segment the target region very well, which shows the effectiveness of the proposed algorithm. 


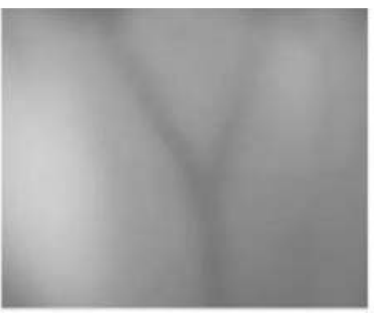

(a)

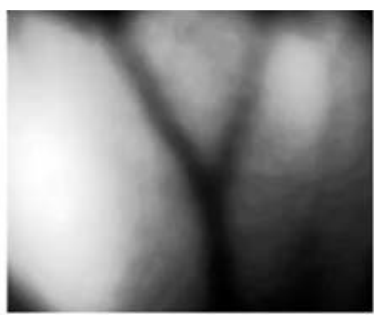

(b)

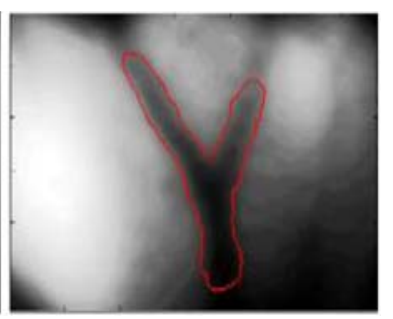

(c)

Figure 1. Image segmentation results of infrared images of dorsal veins of a man's hand: (a) original infrared image; (b) image preprocessing results; and (c) image segmentation results.

In order to verify the effectiveness of the proposed algorithm, this algorithm is applied to the infrared image collected in our laboratory. And this algorithm is compared with the infrared image segmentation algorithm based on Snake model and mathematical morphology method (Snake-MM) proposed in [8]. Figure 2(a) is an image of two hotter objects collected by a laboratory infrared acquisition device which move together. Figure 2(b) is the image segmentation results of the Snake-MM algorithm. Figure 2(c) is the image segmentation results of our algorithm.

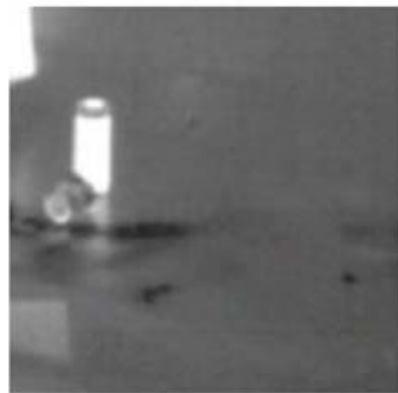

(a)

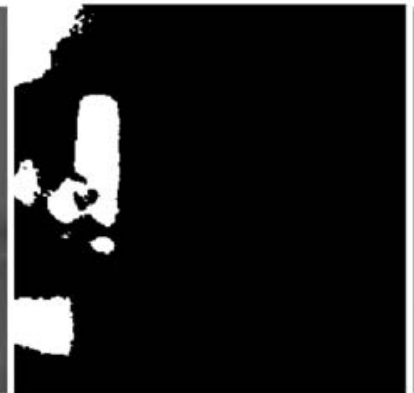

(b)

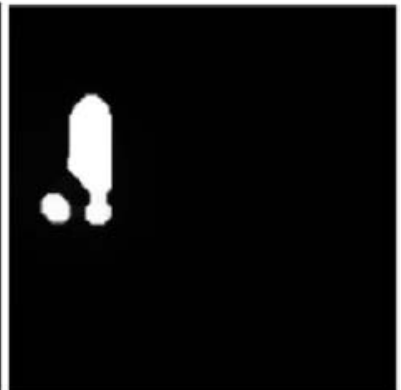

(c)

Figure 2. The infrared image segmentation: (a) the original image; (b) segmentation image by Snake-MM; and (c) segmentation image by ours.

As can be seen from Figure 2(a), there are three targets in the image from an infrared device, where there is a pseudo target in the upper left corner. It is easy to identify and recognize the pseudo target through its 
location. The remaining two targets are stronger on the right and weaker on the left, and the overlap between them will be more pronounced after image enhancement. Ordinary image segmentation algorithms are obviously hard to handle. Figure 2(b) shows that the Snake-MM algorithm cannot remove pseudo targets, and the target segmentation is not complete. There is a certain degree of adhesion between the two targets. Figure 2(c) shows that the proposed algorithm can not only suppress the generation of the pseudo target, but also successfully divide the two targets. This effect is ideal. This fully demonstrates the effectiveness of the proposed algorithm.

\section{Conclusion}

In view of the low contrast of infrared image and the conglutination of targets, etc., an infrared image segmentation algorithm based on local statistical active contour model is proposed in this paper to obtain better image segmentation effect. The algorithm first processes the image, then uses the ACM model to segment the image, and then uses morphological algorithm to deal with the adhesion problem between the segmented object and the interference body. Ultimately we get the final ideal target. Of course, there is still no general method for image segmentation, and the local statistical active contour model has some limitations and shortcomings. Therefore, in the future work, further research will be carried out in this area, and better processing algorithms will be proposed.

\section{Acknowledgement}

This work was supported in part by Natural Science Foundation of China (61401308, 61572063), Natural Science Foundation of Hebei Province (F2016201142, F2016 201187), Science Research Project of Hebei Province (QN2016085, ZC2016040), Science and Technology Support Project of Hebei Province (15210409), Natural Science Foundation

of Hebei University (2014-303), Post-graduate's Innovation Fund Project of Hebei University under grant X201710. 


\section{References}

[1] N. Ning, L. Zhang, D. Zhang et al., Interactive image segmentation by maximal similarity based region merging, Pattern Recognition 43(2) (2010), 445-456.

[2] Y. Boykov and V. Kolmogorov, An experimental comparison of min-cut/max-flow algorithms for energy minimization in vision, IEEE Transactions on Pattern Analysis \& Machine Intelligence 26(9) (2004), 1124-1137.

[3] J. Shi and J. Malik, Normalized cuts and image segmentation, IEEE Transactions on Pattern Analysis \& Machine Intelligence 22(8) (2000), 888-905.

[4] L. A. Vese and T. F. Chan, A multiphase level set framework for image segmentation using the Mumford and Shah model, International Journal of Computer Vision 50(3) (2002), 271-293.

[5] K. Zhang, L. Zhang, H. Song et al., Active contours with selective local or global segmentation: A new formulation and level set method, Image \& Vision Computing 28(4) (2010), 668-676.

[6] R. Guillemaud and M. Brady, Estimating the bias field of MR images, Medical Imaging IEEE Transactions on 16(3) (1997), 238-251.

[7] S. C. Zhu and A. Yuille, Region competition: Unifying snakes region growing and Bayes/MDL for multi-band image segmentation, IEEE Transactions on Pattern Analysis \& Machine Intelligence 18(9) (1996), 884-900.

[8] Xin Qi, Pengfei Li, Shuaiqi Liu et al., Research on the target and interference adhesion problem in the infrared image segmentation, International Journal of Applied Mathematics and Machine Learning 5(1) (2016), 15-24. 